\title{
ON THE ZEROS OF THE SOLUTIONS OF THE EQUATION $w^{(k)}+\left(\operatorname{Re}^{p}+Q\right) w=0$
}

\author{
By Steven B. Bank And J.K. LANGLeY ${ }^{1}$
}

\section{Introduction.}

It was shown in $[3 ; \S 5(\mathrm{~b})$, p. 356$]$ that for any polynomial $P(z)$ of degree $r \geqq 1$, there is a polynomial $Q(z)$ of degree $2 r-2$ such that the equation,

$$
w^{\prime \prime}+\left(e^{P}+Q\right) w=0,
$$

possesses two linearly independent solutions each having no zeros. This result led to an investigation in [4] of the more general equation of arbitrary order $k \geqq 2$,

$$
w^{(k)}+\left(R e^{P}+Q\right) w=0,
$$

where $P, Q$, and $R$ are any polynomials, with $P$ of degree $r \geqq 1$, and $R \not 0$. It was shown in [4] that if the degree of $Q$ is less than $k r-k$, then the exponent of convergence (denoted $\lambda(f)$ ) of the zero-sequence of any solution $f \neq 0$ satisfies $\lambda(f)=\infty$. (We recall (see $\left[12 ;\right.$ p. 250] that if $\left(z_{1}, z_{2}, \cdots\right)$ is the sepuence of zeros of $f(z)$ in $|z|>0$, then $\lambda(f)$ is the infimum of all $\alpha>0$ for which the series $\left|z_{1}\right|^{-\alpha}+\left|z_{2}\right|^{-\alpha}+\cdots$ converges if such an $\alpha$ exists. Otherwise, $\lambda(f)$ is defined to be infinity.)

The above results lead naturally to an investigation of the zero-sequences of solutions of (1.2) in the case when the degree of $Q$ exceeds $k r-k$. One special case has already been successfully treated by the second author in the paper [7] which concerns a problem of $M$. Ozawa [8]. It was shown in [7] that the conclusion $\lambda(f)=\infty$ holds for all solutions $f \not \equiv 0$ of $(1.1)$ when $P$ is linear and $Q$ is nonconstant. In the present paper, we treat the general equation (1.2) of arbitrary order $k \geqq 2$ where the degree of $Q$ exceeds $k r-k$, and we prove that all solutions $f \not \equiv 0$ satisfy $\lambda(f)=\infty$ except possibly when a very special relation exists between $P$ and $Q$. Our main result is :

THEOREM 1.1. Let $k$ be an integer greater than 1 , and let $P, Q$, and $R$ be polynomials with $R \neq 0, \operatorname{deg}(P)=r \geqq 1$, and $\operatorname{deg}(Q)=n>k r-k$, say $Q(z)=a_{n} z^{n}+\cdots$, and $P(z)=b_{r} z^{r}+\cdots$. Let $c_{1}, \cdots, c_{k}$ be the distinct roots of the equation $c^{k}+a_{n}=0$,

This research was supported in part by the National Science Foundation (DMS-8721813). Received August 4, 1989; Revised February 21, 1990. 
and assume that there exists a real number $\theta_{0}$ in $(-\pi, \pi]$ for which,

$$
\cos \left(r \theta_{0}+\arg b_{r}\right)=0
$$

and

$$
\cos \left(((n+k) / k) \theta_{0}+\arg c_{\jmath}\right) \neq 0 \quad \text { for } \quad j=1, \cdots, k .
$$

Then the exponent of convergence of the zero-sequence of any solution $f \neq 0$ of (1.2) is infinite.

We remark that in the exceptional case when no such point $\theta_{0}$ exists satisfying (1.3) and (1.4) simultaneously, the situation concerning $\lambda(f)$ is still unclear, but the authors conjecture that the conclusion $\lambda(f)=\infty$ is still valid for all solutions $f \neq 0$. However, our proof will not handle this special case for the following reason: A ray $\arg z=\theta_{0}$ for which (1.3) holds represents a dividing line between two adjoining sectors where the growth of $e^{P}$ is changing from very fast to very small. For a solution $f \not \equiv 0$ with $\lambda(f)<\infty$, we can obtain extensive information on the form of $f$ in the sector where $e^{P}$ is growing fast by using certain techniques from Nevanlinna theory (notably Clunie's lemma (see $\left[6 ;\right.$ p. 68])). In the adjoining sector where $e^{P}$ is decaying, the equation (1.2) is approximated by the equation, $w^{(k)}+Q w=0$, and this fact allows us to asymptotically integrate (1.2) in this sector to yield a fundamental set (see $\S 3$ below). The condition (1.4) is needed in our proof to ensure that the dominant terms in this fundamental set have the same growth pattern in both of the adjoining sectors thus providing a "connection" between the two sectors.

Finally, the authors would like to thank the referee for valuable suggestions to improve the readability of the paper.

\section{Preliminaries.}

(A). [5; pp. 11-12]. An $R$-set is a countable union of disks whose centers converge to $\infty$, and whose radii have finite sum. The set of all real $\theta$ for which the ray $z=r e^{\imath \theta}$ meets infinitely many disks in an $R$-set, has measure zero.

(B). Let $f(z)$ be a meromorphic function on the plane. If $H(f)$ is a polynomial in $f, f^{\prime}, f^{\prime \prime}, \cdots, f^{(k)}$, whose coefficients are meromorphic functions, say

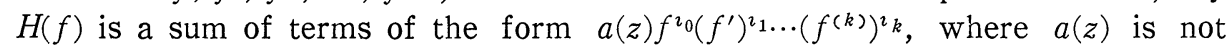
identically zero, then the maximum of all the numbers, $i_{0}+i_{1}+\cdots+i_{k}$ in the terms of $H(f)$, is called the total degree of $H(f)$. In addition, we use the standard notation (see $[6 ;$ p. 4]) for the Nevanlinna proximity function $m(r, f)$ of the function $f(z)$, which is defined as $m(r, f)=(2 \pi)^{-1} \int_{0}^{2 \pi} \log ^{+}\left|f\left(r e^{2 \theta}\right)\right| d \theta$, where $\log ^{+} x$ denotes the maximum of $\log x$ and zero. We will need the following slight variant of Clunie's lemma [6; p. 68]. The proof is identical to the proof in $[6]$. 
LEMMA 2.1. Let $n$ be a positive integer, and let $f$ be a transcendental, meromorphic solution of the equation $f^{n} G(f)=H(f)$, where $G(f)$ and $H(f)$ are polynomials in $f, f^{\prime}, \cdots, f^{(k)}$ (for some $k \geqq 1$ ), whose coefficients are meromorphic functions. Let $\Delta$ denote the set of all coefficients, and assume that the total degree of $H(f)$ is at most $n$. Then,

$$
m(r, G(f))=0\left(\Psi(r)+1+\sum_{j=1}^{k} m\left(r, f^{(j)} / f\right)\right) \text { as } r \longrightarrow \infty \text {, }
$$

where $\Psi(r)=\max \{m(r, a): a \in \Delta\}$.

(C). We will need the following concepts from [11]:

(a) $[11: \S 94]$ : The neighborhood system $F(a, b)$. Let $-\pi \leqq a<b \leqq \pi$. For each nonnegative real-valued function $g$ on $(0 .(b-a) / 2)$, let $V(g)$ be the union (over all $\delta \in(0 .(b-a) / 2)$ ) of all sectors, $a+\delta<\arg (z-h(\delta))<b-\delta$, where $h(\delta)=$ $g(\delta) e^{\imath(a+b) / 2}$. The set of all $V(g)$ (for all choices of $g$ ) is denoted $F(a, b)$, and is a filter base which converges to $\infty$. Each $V(g)$ is a simply-connected region (see $[11 ; \S 93]$ ), and we require the following simple fact which is proved in [2; p. 74]:

LEMMA 2.2. Let $V$ be an element of $F(a, b)$, and let $\varepsilon>0$ be arbitrary. Then there is a constant $R_{0}(\varepsilon)>0$ such that $V$ contains the set, $a+\varepsilon \leqq a r g z \leqq b-\varepsilon$, $|z| \geqq R_{0}(\varepsilon)$.

As in [1], we will say that a statement holds "except in finitely many directions in $F(a, b)$ ", if there exist finitely many points $r_{1}<r_{2}<\cdots<r_{q}$ in $(a, b)$ such that the statement holds in each of $F\left(a, r_{1}\right), F\left(r_{1}, r_{2}\right), \cdots, F\left(r_{q}, b\right)$ separately.

(b) $[11: \S 13]$ : The relation of asymptotic equivalence. If $f(z)$ is an analytic function on some element of $F(a, b)$, then $f(z)$ is called admissible in $F(a, b)$. If $c$ is a complex number, then the statement $f \rightarrow c$ in $F(a, b)$ means (as is customary) that for any $\varepsilon>0$, there exists an element $V$ of $F(a, b)$ such that $|f(z)-c|<\varepsilon$ for all $z \in V$. The statement $f \ll 1$ in $F(a, b)$, means that in addition to $f \rightarrow 0$, all the functions $\theta_{j}^{k} f \rightarrow 0$ in $F(a, b)$, where $\theta$, denotes the operator $\theta_{\jmath} f=z(\log z) \cdots\left(\log _{\jmath_{-1}} z\right) f^{\prime}(z)$, and where (for $\left.k \geqq 0\right), \theta_{\jmath}^{k}$ is the kth iterate of $\theta_{\jmath}$. The statements $f_{1} \ll f_{2}$ and $f_{1} \sim f_{2}$ in $F(a, b)$ mean respectively $f_{1} / f_{2} \ll 1$ and $f_{1}-f_{2} \ll f_{2}$. (This strong relation of asymptotic equivalence is designed to ensure that if $M$ is a non-constant logarithmic monomial of rank $\leqq p$ (i.e. a function of the form,

$$
M(z)=K z^{a_{0}}(\log z)^{a_{1}} \cdots\left(\log _{p} z\right)^{a} p,
$$

for real $a_{\jmath}$, and complex $K \neq 0$ ), then $f \sim M$ implies $f^{\prime} \sim M^{\prime}$ in $F(a, b)$ (see $[11$ : $\S 28]$ ). As usual, $z^{\alpha}$ and $\log z$ will denote the principal branches of these functions on $|\arg z|<\pi$.) We will write $f_{1} \approx f_{2}$ to mean $f_{1} \sim c f_{2}$ for some nonzero constant $c$. An admissible function $f(z)$ in $F(a, b)$ is called trivial in $F(a, b)$ if $f \ll z^{-\alpha}$ in $F(a, b)$ for every $\alpha>0$. If $f \sim c z^{-1+d}$ in $F(a, b)$, where $c \neq 0$ and 
$d>0$, then the indicial function of $f$ is the function.

$$
I F(f, \varphi)=\cos (d \varphi+\arg c) \quad \text { for } a<\varphi<b .
$$

If $g$ is any admissible function in $F(a, b)$, we will denote by $\int g$, any primitive of $g$ in an element of $F(a, b)$. We will require the following fact (see [2; p. 75]):

LEMMA 2.3. Let $f \sim c z^{-1+d}$ in $F(a, b)$, where $c \neq 0$ and $d>0$. If $\left(a_{1}, b_{1}\right)$ is any subinterval of $(a, b)$ on which $\operatorname{IF}(f, \phi)<0$ (respectively, $\operatorname{IF}(f, \phi)>0)$, then for all real $\alpha, \exp \int f \ll z^{\alpha}$ (respectively, $\left.\exp \int f \gg z^{\alpha}\right)$ in $F\left(a_{1}, b_{1}\right)$.

(c) The operator $\theta_{1}$ defined by $\theta_{1} f=z f^{\prime}$, will be denoted simply $\theta$. It is easy to prove by induction that for each $n=1,2, \cdots$

$$
f^{(n)}=z^{-n}\left(\sum_{j=1}^{n} b_{j n} \theta^{\jmath} f\right) \text {. }
$$

(d) [11: \$49]. A logarithmic domain of rank zero (briefly, an $L D_{0}$ ) over $F(a, b)$ is a complex vector space $L$ of admissible functions in $F(a, b)$, which contains the constants, and such that any finite linear combination of elements of $L$, with coefficients which are logarithmic monomials of rank $\leqq p$ for some $p \geqq 0$, is either trivial in $F(a, b)$ or is $\sim$ to a logarithmic monomial of rank $\leqq p$ in $F(a, b)$. (The simplest examples of such sets $L$ (where we can take $(a, b)$ to be any open subinterval of $(-\pi, \pi)$ ) are the set of all polynomials, the set of all rational functions, and the set of all rational combinations of logarithmic monomials of rank $\leqq 0$. More extensive examples can be found in [11: §§ 128, 53].)

$(e)[1 ; \S 3]$. If $G(v)$ is a polynomial in $v$, whose coefficients belong to an $L D_{0}$ over $F(a, b)$, then a logarithmic monomial $M$ is called a critical monomial of $G$ if there exists an admissible function $h \sim M$ in $F(a, b)$ such that $G(h)$ is not $\sim G(M)$ in $F(a, b)$. The set of critical monomials of $G$ can be produced by using the algorithm in $[10 ;$ p. 236] which is based on a Newton polygon construction. This algorithm shows that the critical monomials are of rank $\leqq 0$. (In the special case where the coefficients of $G(v)$ are rational functions, the critical monomials are precisely the functions $c z^{\alpha}$ which form the first term of one of the expansions around $z=\infty$ of the algebraic function defined by $G(v)=0$. This fact follows from $[10 ; \S 26])$.) Associated with a critical monomial $M$ of $G(v)$ is a positive integer which is called its multiplicity. The multiplicity is defined as the smallest positive integer $j$ such that $M$ is not a critical monomial of $\partial^{\jmath} G / \partial v^{\jmath}$. (See $[10 ;$ p. 231]). If the multiplicity is equal to 1 , the critical monomial $M$ is called simple.

(D). We will need the following concepts from [1] and [9]:

(a). Let $n$ be a positive integer, and let $\left\{R_{0}(z), \cdots, R_{n}(z)\right\}$ be contained in an $L D_{0}$ over $F(a, b)$ for some $(a, b)$ with $-\pi \leqq a<b \leqq \pi$, and assume that $R_{n}(z)$ 
is non-trivial (see $\S 2(C)$ in $F(a, b)$. Using (2.4), rewrite the equation,

$$
R_{n}(z) w^{(n)}+R_{n-1}(z) w^{(n-1)}+\cdots R_{0}(z) w=0,
$$

in the form,

$$
\Omega(w)=\sum_{j=0}^{n} B_{j}(z) \theta^{\jmath} w=0, \quad \text { where } \quad \theta^{0} w=w .
$$

By dividing equation (2.6) through by the highest power of $z$ which occurs in the expansions of all the functions $B_{j}(z)$ for all $j=0, \cdots, n$, we may assume that for each $j$, we have either $B_{j} \ll 1$ or $B_{j} \approx 1$ in $F(a, b)$, and there exists an integer $p \geqq 0$ such that $B_{j} \ll 1$ for $j>p$, while $B_{p}$ is $\sim$ to a nonzero constant (denoted $B_{p}(\infty)$ ). The integer $p$ is called the critical degree of the equation (2.5). The equation,

$$
F^{*}(\alpha)=\sum_{j=0}^{n} B_{j}(\infty) \alpha^{\jmath}=0,
$$

is called the critical equation of (2.5). Clearly, $F^{*}(\alpha)$ is a polynomial in $\alpha$, of degree $p$, having constant coefficients.

When (2.5) is written in the form (2.6) we form the algebraic polynomial in $v$,

$$
H(v)=\sum_{j=0}^{n} z^{\jmath} B_{j}(z) v^{\jmath},
$$

which we will call the full factorization polynomial for (2.5).

Let $W$ belong to an $L D_{0}$ over $F(a, b)$, and assume that $W \gg z^{-1}$ in $F(a, b)$. Set $h=\exp \int W$, and let $L(v)$ be the operator defined by $L(v)=\Omega(h v) / h$. Let $H(u)$ and $K(u)$ denote respectively, the full factorization polynomials for $\Omega(w)$ and $L(v)$. In $[9 ; \S 10]$, the following concept is introduced: $W$ is said to have transform type $(m, q)$ with respect to $H$ (briefly, $\operatorname{trt}(W, H)=(m, q))$ if $L(v)=0$ has critical degree $m$, and if $q$ is the minimum multiplicity of all critical monomials $M$ of $K(u)$ which satisfy $z^{-1} \ll M \ll W$ in $F(a, b)$. (If there are no such $M$, then we set $q=0$.)

\section{We will require the following lemma on asymptotic integration.}

LEMMA 3.1. Let $k$ be a positive integer, and let $Q(z)$ be a rational function whose expansion around $\infty$ is $Q(z)=a_{n} z^{n}+a_{n-1} z^{n-1}+\cdots$, where $a_{n} \neq 0$ and $n / k>$ -1 . Let $c_{1}, \cdots, c_{k}$ be the distinct roots of the equation $c^{k}+a_{n}=0$. Let $(a, b)$ be any open subinterval of $(-\pi, \pi)$, and let $G(z)$ be an analytic function in an element of $F(a, b)$ which satisfies $G \ll z^{\beta}$ over $F(a, b)$, for every real number $\beta$. Then, for each integer $j$ in $\{1, \cdots, k\}$, there exist a complex number $\alpha$, and a function $E$, which is analytic in an element of $F(-\pi, \pi)$, which have the following two properties: 
(a) $E_{j} \sim c_{j} z^{n / k}$ over $F(-\pi, \pi)$;

(b) Except in finitely many directions in $F(a, b)$, the equation, $w^{(k)}+(Q+$ $G) w=0$, possesses a fundamental set of solutions $\varphi_{1}, \cdots, \varphi_{k}$, where each $\varphi_{j}$ is of the form $\varphi_{j}=h, \exp \int E$, for some analytic function $h$, satisfying $h_{j} \sim z^{\alpha}$.

Remark. (1) The proof will show that each function $E$, is actually a single-valued branch of an algebraic function (which depends on $c_{\jmath}$ ).

(2) As will be seen from the proof, the lemma will hold if the hypothesis that $Q$ is rational is replaced by the assumption that in some element of $F(-\pi, \pi)$, the function $Q$ is a single-valued analytic branch of an algebraic function, and satisfies $Q \sim a_{n} z^{n}$ in $F(-\pi, \pi)$ for some complex $a_{n} \neq 0$, and some rational number $n>-k$.

\section{Proof of Lemma 3.1.}

We will apply results from [1] and [9] to the operator $L(w)=w^{(k)}+Q w$. For this purpose, we rewrite the operator $L$ in terms of the operator $\theta$ defined by $\theta w=z w^{\prime}$ (and $\theta^{2} w=\theta(\theta w)$ etc.). It is easy to see that we obtain

$$
L(w)=z^{-k}(\theta(\theta-1) \cdots(\theta-k+1) w)+Q w .
$$

Following $\S 2(\mathrm{D})$, we associate with $L$ the full factorization polynomial $H(v)$ defined by,

$$
H(v)=z^{-k}(z v(z v-1) \cdots(z v-k+1))+Q .
$$

In view of the expansion of $Q$ around $\infty$, it is clear that each function $N_{j}=$ $c_{j} z^{n / k}$ is the first term of one of the $k$ expansions around $\infty$ of the algebraic function defined by $H(v)=0$. (In the terminology of $\S 2(\mathrm{C})$, the functions $N$, are the critical monomials of $H(v)$ and each is simple.) Using the terminology of $\S 2(D)$, it follows from [9; Lemmas $10.3,10.5]$ that for each $j$, there is an analytic function $E$, satisfying Part $(a)$ of the lemma and having the property that its transform type with respect to $H(v)$ is $(1,0)$. This means that the operator $L_{j}(u)$ defined by

$$
L_{j}(u)=\left(\exp \left(-\int E_{\jmath}\right)\right) L\left(\left(\exp \int E_{\jmath}\right) u\right)
$$

has critical degree 1 in the terminology of $\S 2(D)$, and therefore from $[1 ; p$. 147, Formulas $(a),(b)]$, there is a real number $t$ such that when the operator $z^{-t} L_{j}(u)$ is written in terms of $\theta$, say

$$
z^{-t} L_{j}(u)=\sum_{m=0}^{k} B_{m}(z) \theta^{m} u
$$

the following asymptotic relations hold over $F(-\pi, \pi)$ : For each $m>1$, we have $B_{m} \ll 1$; there is aneonzero constant $K_{1}$ such that $B_{1} \sim K_{1}$; either $B_{0} \ll 1$ or 
$B_{0} \sim K_{0}$ for some nonzero constant $K_{0}$.

We now define $M(w)=w^{(k)}+(Q+G) w$, and as above define,

$$
M_{j}(u)=\left(\exp \left(-\int E_{\jmath}\right)\right) M\left(\left(\exp \int E_{\jmath}\right) u\right)
$$

Since $M(w)=L(w)+G w$, we have,

$$
z^{-t} M_{j}(u)=z^{-t} L_{j}(u)+z^{-t} G u=\sum_{m=0}^{k} D_{m} \theta^{m} u,
$$

where $D_{m}=B_{m}$ for $m>0$, and $D_{0}=B_{0}+z^{-t} G$. In view of the hypothesis that $G \ll z^{\beta}$ over $F(a, b)$ for every real $\beta$, it follows from [11; Lemma 53(c)] that with $F(a, b)$ as the neighborhood system, the operator $z^{-t} M_{j}(u)$ is of the type treated in [1] (i. e. the coefficients belong to a logarithmic domain of rank zero over $F(a, b))$. In addition, the asymptotic relations for the $B_{m}$ listed above show that the critical degree of $z^{-t} M_{j}(u)$ is 1 . Thus if $\alpha$, is the root of the critical equation (see $[1 ; \S 3(\mathrm{e})]$ ) for $z^{-t} M_{j}(u)$, then it follows from $[1$; Lemma 5 and Theorem 1] that except in finitely many directions in $F(a, b)$, the equation $z^{-t} M_{j}(u)=0$ possesses a solution $h_{j} \sim z^{\alpha}$. Thus, such functions $\varphi_{\jmath}=h, \exp \int E_{\jmath}$ for $j=1, \cdots, k$, exist except in finitely many directions in $F(a, b)$, and are solutions of $w^{(k)}+(Q+G) w=0$. The fact that they are linearly independent

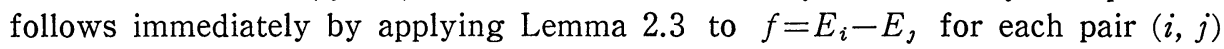
with $i \neq j$.

\section{Proof of Theorem 1.1.}

We observe first that we can assume that $\theta_{0}$ belongs to $(-\pi, \pi)$, since if $\theta_{0}=\pi$, then the change of variable $\rho=-z$ in (1.2) transforms (1.2) into an equation in $\rho$ for which the hypothesis is satisfied for $\theta_{0}=0$.

We assume contrary to the conclusion that (1.2) possesses a solution $f \not \equiv 0$ satisfying $\lambda(f)<\infty$. Thus we may write $f=G e^{h}$, where $G$ and $h$ are entire functions with $G$ of finite order. Thus, from (1.2) we obtain,

$$
\left(h^{\prime}\right)^{k}+\Phi_{k-1}\left(h^{\prime}\right)+R e^{P}+Q=0,
$$

where $\Phi_{k-1}\left(h^{\prime}\right)$ is a differential polynomial of total degree at most $k-1$ in $h^{\prime}, h^{\prime \prime}, \cdots$, whose coefficients are polynomials in $G^{\prime} / G, G^{\prime \prime} / G, \cdots, G^{(k)} / G$ having constant coefficients. Applying Lemma 2.1 to (5.1), we find that $h^{\prime}$ is of finite order.

Now set

$$
S=h^{\prime \prime}-\left(\left(R P^{\prime}+R^{\prime}\right) / k R\right) h^{\prime} .
$$

We now differentiate (5.1) and subtract this new expression from the expression obtained from (5.1) by multiplying it by $\left(R P^{\prime}+R^{\prime}\right) / R$. The terms in $e^{P}$ cancel and we obtain 


$$
\left(h^{\prime}\right)^{k-1}(-k S)=\Psi_{k-1}\left(h^{\prime}\right),
$$

where $\Psi_{k-1}\left(h^{\prime}\right)$ is a differential polynomial of total degree at most $k-1$ whose coefficients are all meromorphic functions $\phi$ satisfying $m(r, \phi)=0(\log r)$ as $r \rightarrow \infty$. Since $h^{\prime}$ is of finite order, it follows from Lemma 2.1 that $m(r, S)=0(\log r)$ as $r \rightarrow \infty$. However, since $R$ is a polyromial, we see from (5.2) that $S$ can have only finitely many poles. Thus $S$ is a rational function.

We now consider the equation,

$$
w^{\prime}-\left(\left(R P^{\prime}+R^{\prime}\right) / k R\right) w=S .
$$

If $S \equiv 0$, set $w_{0}(z) \equiv 0$. If $S \equiv 0$, it follows from [11; Theorem III, Part (b), p. 72] that there exist $\varepsilon_{1}>0$ and an analytic solution $w_{0}(z)$ of $(5.4)$ in an element of $F\left(\theta_{0}-\varepsilon_{1}, \theta_{0}+\varepsilon_{1}\right)$, such that,

$$
w_{0} \sim-k S / P^{\prime} \text { over } F\left(\theta_{0}-\varepsilon_{1}, \theta_{0}+\varepsilon_{1}\right) .
$$

(Thus, in both of the cases, $S \equiv 0$ and $S \not \equiv 0$, the function $w_{0}$ solves (5.4).)

Now, let $D(z)$ denote a single-valued analytic branch of $R^{1 / k}$ on an element of $F(-\pi, \pi)$. (Thus clearly, if $R(z)=t_{p} z^{p}+\cdots$, then $D \sim t_{p}^{1 / k} z^{p / k}$ over $F(-\pi, \pi)$, for some choice of $t_{p}^{1 / k}$.). In view of (5.2) and (5.4), it easily follows that for some constant $K_{1}$, we have

$$
h^{\prime}=w_{0}+K_{1} D e^{P / k} \quad \text { in some element of } F\left(\theta_{0}-\varepsilon_{1}, \theta_{0}+\varepsilon_{1}\right) \text {. }
$$

Now the terms of total degree $k-1$ in the differential polynomial $\Phi_{k-1}\left(h^{\prime}\right)$ in (5.1) are easily seen to be,

$$
k\left(h^{\prime}\right)^{k-1}\left(G^{\prime} / G\right)+(k(k-1) / 2)\left(h^{\prime}\right)^{k-2} h^{\prime \prime} .
$$

Hence, when (5.6) is substituted into (5.1), we obtain an equation,

$$
\sum_{m=0}^{k} \Omega_{m}(z) e^{m P(z) / k} \equiv 0,
$$

where,

$$
\begin{aligned}
& \Omega_{k}=K_{1}^{k} D^{k}+R=R\left(K_{1}^{k}+1\right), \\
& \Omega_{k-1}=k K_{1}^{k-1} D^{k-1} V,
\end{aligned}
$$

where,

$$
V=w_{0}+\left(G^{\prime} / G\right)+((k-1) / 2)\left(\left(P^{\prime} / k\right)+\left(D^{\prime} / D\right)\right),
$$

and where for each $m$, the function $\Omega_{m}(z)$ is a polynomial (with constant coefficients) in the variables $G^{\prime} / G, \cdots, G^{(k)} / G, w_{0}, w_{0}^{\prime}, \cdots, D, D^{\prime}, \cdots$, and $Q, P^{\prime}, P^{\prime \prime}, \cdots$. Standard estimates on the logarithmic derivative of an entire function of finite order, together with the known asymptotic behavior of $w_{0}$ 
and $D$ developed earlier (and the remarks in Parts (A), (B), and (C) in $\S 2$ ), yield a real number $N>0$ with the following property: For all $\theta$ in $\left(\theta_{0}-\varepsilon_{1}, \theta_{0}+\varepsilon_{1}\right)$ outside a set of zero measure, we have for all $m=0,1, \cdots, k$,

$$
\left|\Omega_{m}\left(r e^{2 \theta}\right)\right|=0\left(r^{N}\right) \quad \text { as } r \rightarrow+\infty .
$$

Noting that the left side of (1.3) is simply the indicial function $\operatorname{IF}\left(P^{\prime}, \varphi\right)$ evaluated at $\varphi=\theta_{0}$, (see (2.3)) we can choose $\varepsilon_{2}>0$ so small that $\varepsilon_{2}<\varepsilon_{1}$ and such that the only zero of $\operatorname{IF}\left(P^{\prime}, \varphi\right)$ on $\left(\theta_{0}-\varepsilon_{2}, \theta_{0}+\varepsilon_{2}\right)$ is at $\varphi=\theta_{0}$. Since $\operatorname{IF}\left(P^{\prime}, \varphi\right)$ must change sign at $\varphi=\theta_{0}$, this function has constant signs on $\left(\theta_{0}-\varepsilon_{2}, \theta_{0}\right)$ and $\left(\theta_{0}, \theta_{0}+\varepsilon_{2}\right)$, and these signs are different. Without loss of generality we can assume that

$$
\operatorname{IF}\left(P^{\prime}, \varphi\right)>0 \text { on }\left(\theta_{0}-\varepsilon_{2}, \theta_{0}\right)
$$

and

$$
\operatorname{IF}\left(P^{\prime}, \varphi\right)<0 \text { on }\left(\theta_{0}, \theta_{0}+\varepsilon_{2}\right),
$$

since the argument will be symmetric if we interchange the two intervals.

Now from (5.13) and Lemma 2.3, we see that for $m=1, \cdots, k$, we have $e^{-m P / k} \ll z^{\beta}$ for all real $\beta$, over $F\left(\theta_{0}-\varepsilon_{2}, \theta_{0}\right)$. Thus, if we divide the equation (5.8) by $e^{P}$, and evaluate the resulting relation on any ray $\arg z=\theta$, where $\theta$ is an element of $\left(\theta_{0}-\varepsilon_{2}, \theta_{0}\right)$ for which (5.12) is valid, we see that $\Omega_{k}\left(r e^{i \theta}\right)=o\left(r^{\beta}\right)$ for all real $\beta$ as $r \rightarrow+\infty$. Since $R$ in (5.9) is a polynomial which is not identically zero, we must have $K_{1}^{k}=-1$. Thus, $\Omega_{k}(z) \equiv 0$. We now divide the relation (5.8) by $e^{(k-1) P / k}$, and as above we find that for all $\theta$ in $\left(\theta_{0}-\varepsilon_{2}, \theta_{0}\right)$, with the possible exception of a set $H$ of zero measure where (5.12) fails to hold, we have $\Omega_{k-1}\left(r e^{i \theta}\right)=o\left(r^{\beta}\right)$ for all real $\beta$ as $r \rightarrow+\infty$. Since $K_{1}$ is a nonzero constant, and $D$ in (5.10) satisfies $D \sim t_{p}^{1 / k} z^{p / k}$ over $F(-\pi, \pi)$ for certain constants $p$ and $t_{p}$, it follows from (5.10) that $V(z)$ has the property that for all $\theta$ in $\left(\theta_{0}-\varepsilon_{2}, \theta\right)_{0}-H$, we have

$$
V\left(r e^{i \theta}\right)=o\left(r^{\beta}\right) \text { for all real } \beta \text { as } r \rightarrow+\infty \text {. }
$$

In view of the asymptotic behavior of $D$, clearly there exists an analytic branch $D_{1}$ of $D^{(k-1) / 2}$ in an element $T$ of $F\left(\theta_{0}-\varepsilon_{2}, \theta_{0}+\varepsilon_{2}\right)$, and we can assume that $w_{0}(z)$ is also analytic in $T$. We now define a function $W(z)$ on $T$ by,

$$
W=G D_{1} e^{(k-1) P / 2 k} e^{\int w_{0}},
$$

where $\int w_{0}$ represents a fixed primitive of $w_{0}$ on $T$. In view of the asymptotic behavior of $D$ and $w_{0}$ (see (5.5)) and the fact that $G$ is of finite order, it easily follows (using Lemma 2.2) that if we set $\varepsilon_{3}=\varepsilon_{2} / 2$, then there is a constant $r_{0}>0$ such that $W$ is analytic and of finite order in the region,

$$
\theta_{0}-\varepsilon_{3}<\arg z<\theta_{0}+\varepsilon_{3}, \quad|z|>r_{0} .
$$


It is clear that $W^{\prime} / W=V$ on $T$, and hence from (5.15) it easily follows that for each $\theta$ in $\left(\theta_{0}-\varepsilon_{3}, \theta_{0}\right)-H$, there is a nonzero constant $J_{\theta}$ such that $W\left(r e^{i \theta}\right) \rightarrow J_{\theta}$ as $r \rightarrow+\infty$. It follows from Phragmen-Lindelof principles $[12 ; \S 5.61,5.64$, pp. 176-180] that all $J_{\theta}$ are equal to a single number $J$, and we have,

$$
W\left(r e^{i \theta}\right) \rightarrow J \neq 0 \text { as } r \rightarrow+\infty \text { for } \theta_{0}-\varepsilon_{3}<\theta<\theta_{0} .
$$

Since $f=G e^{h}$, it follows from (5.6) and (5.16) that we can write on the domain $T$,

$$
\begin{aligned}
& W=\phi f D_{1} e^{(k-1) P / 2 k}, \quad \text { where } \\
& \phi=K_{2} \exp \left(-K_{1} \int D e^{P / k}\right),
\end{aligned}
$$

for some constant $K_{2} \neq 0$ and some fixed choice of the primitive in (5.20). In view of (5.14) and Lemma 2.3, we see that $-K_{1} D e^{P / k} \ll z^{\beta}$ for all real $\beta$, over $F\left(\theta_{0}, \theta_{0}+\varepsilon_{2}\right)$, and it immediately follows from $[2 ; \S 2$, Lemma B, Part (b)] that for some constant $K_{3} \neq 0$, we have

$$
\phi \rightarrow K_{3} \text { over } F\left(\theta_{0}, \theta_{0}+\varepsilon_{2}\right) .
$$

Again in view of (5.14) and Lemma 2.3, we see that in $F\left(\theta_{0}, \theta_{0}+\varepsilon_{2}\right)$, the function $R e^{P}$ satisfies the hypothesis for $G$ in Lemma 3.1. Hence by Lemma 3.1 , there exists $\varepsilon_{4}$ in $\left(0, \varepsilon_{3}\right)$ such that in some element of $F\left(\theta_{0}, \theta_{0}+\varepsilon_{4}\right)$, the equation (1.2) possesses a fundamental set of solutions $\varphi_{\jmath}=h_{\jmath} \exp \int E_{\jmath}$ for $j=1, \cdots, k$, where

$$
\begin{aligned}
& E_{j} \sim c_{\jmath} z^{n / k} \text { over } F(-\pi, \pi), \text { and } \\
& h_{j} \sim z^{\alpha} \text { over } F\left(\theta_{0}, \theta_{0}+\varepsilon_{4}\right) \text { for some constant } \alpha_{\jmath} .
\end{aligned}
$$

Hence, we can write $f$ as a linear combination of $\varphi_{1}, \cdots, \varphi_{k}$, say $f=\Sigma \beta_{j} \varphi_{\jmath}$. Since some $\beta, \neq 0$ (since $f \not \equiv 0$ ), the set $I$ consisting of all $j$ in $\{1, \cdots, k\}$ for which $\beta_{j} \neq 0$, is not empty. Since the constants $c_{j}$ in (5.22) are distinct, the union of the sets of zeros on $(-\pi, \pi)$ of the functions $\operatorname{IF}\left(E_{i}-E_{\jmath}, \theta\right)$ for all pairs $(i, j)$ in $I \times I$ with $i \neq j$, is a finite set. Letting $\varepsilon_{5}>0$ be so small that $\left(\theta_{0}, \theta_{0}+\varepsilon_{5}\right)$ contains none of these zeros, it follows from Lemma 2.3 (applied to $f=E_{i}-E_{\jmath}$ ) that for each pair $(i, j)$ in $I \times I$ with $i \neq j$, either $\varphi_{i} \ll \varphi_{j}$ or $\varphi_{j} \ll \varphi_{i}$ in $F\left(\theta_{0}, \theta_{0}+\varepsilon_{5}\right)$. Thus, there exists an element $m$ in $I$ such that $\varphi_{j} \ll \varphi_{m}$ in $F\left(\theta_{0}, \theta_{0}+\varepsilon_{5}\right)$ for all $j$ in $I$ with $j \neq m$. Hence we have,

$$
f=\beta_{m} \varphi_{m}(1+E) \quad \text { where } E \rightarrow 0 \text { over } F\left(\theta_{0}, \theta_{0}+\varepsilon_{5}\right) .
$$

Now for $j=m$, the left side of (1.4) is $\operatorname{simply} \operatorname{IF}\left(E_{m}, \theta\right)$ evaluated at $\theta=\theta_{0}$, and is nonzero by hypothesis. Hence we can assume that $\varepsilon_{5}$ chosen above is so small that 


$$
\operatorname{IF}\left(E_{m}, \theta\right) \text { is nowhere zero on }\left(\theta_{0}-\varepsilon_{5}, \theta_{0}+\varepsilon_{5}\right) \text {. }
$$

Now, in view of (5.19), (5.21), (5.24), and the definition of $D_{1}$, we can write,

$$
W=\phi_{1} h_{m}(1+E) \exp \int U \text { on } F\left(\theta_{0}, \theta_{0}+\varepsilon_{5}\right),
$$

where

$$
U=E_{m}+((k-1) / 2 k) P^{\prime}+((k-1) / 2)\left(D^{\prime} / D\right),
$$

and where for some constant $K_{4} \neq 0$,

$$
\phi_{1} \rightarrow K_{4} \neq 0 \text { over } F\left(\theta_{0}, \theta_{0}+\varepsilon_{5}\right) .
$$

Since $D$ is a branch of an algebraic function and satisfies $D \sim t_{p}^{1 / k} z^{p / k}$ over $F(-\pi, \pi)$, it follows from $\S 2(C)$ that either $D^{\prime} / D \ll z^{-1}$ or $D^{\prime} / D \sim(p / k) z^{-1}$ over $F(-\pi, \pi)$. In addition, by hypothesis, we have $n / k>r-1$ where $r$ is the degree of $P$, and hence from (5.22), we see that

$$
U \sim E_{m} \text { over } F(-\pi, \pi) .
$$

We now distinguish the two cases given by (5.25).

Case I. $\operatorname{IF}\left(E_{m}, \theta\right)<0$ on $\left(\theta_{0}-\varepsilon_{5}, \theta_{0}+\varepsilon_{5}\right)$. In this case, it follows from (5.29) and Lemma 2.3 that $\exp \int U \ll z^{\beta}$ for all real $\beta$, over $F\left(\theta_{0}-\varepsilon_{5}, \theta_{0}+\varepsilon_{5}\right)$. Hence, in view of the representation (5.26) (and the asymptotic relations (5.23), (5.24) and (5.28)), we see that $W \rightarrow 0$ over $F\left(\theta_{0}, \theta_{0}+\varepsilon_{5}\right)$. This fact, together with (5.18) provides a contradiction of the Phragmen-Lindelof principle $[12 ; \S 5.64$, p. 179].

Case II. $\operatorname{IF}\left(E_{m}, \theta\right)>0$ on $\left(\theta_{0}-\varepsilon_{5}, \theta_{0}+\varepsilon_{5}\right)$. In this case, we define,

$$
W_{0}=z^{-\alpha_{m}} W \exp \left(-\int U\right) \text {. }
$$

Since $W$ is analytic on the region (5.17), and since $z^{-\alpha_{m}}$ and $\exp \left(-\int U\right)$ are analytic on an element of $F(-\pi, \pi)$, clearly $W_{0}$ is analytic on the region (5.17) (possibly with a larger $r_{0}$ ). Since $W$ is of finite order on the region (5.17), the same is clearly true of $W_{0}$ (by (5.22) and (5.29)). From Lemma 2.3, we have that $\exp \left(-\int U\right) \ll z^{\beta}$ for all real $\beta$ over $F\left(\theta_{0}-\varepsilon_{5}, \theta_{0}+\varepsilon_{5}\right)$ and so from (5.18), we see that

$$
W_{0}\left(r e^{i \theta}\right) \rightarrow 0 \text { as } r \rightarrow+\infty \text { for } \theta_{0}-\varepsilon_{5}<\theta<\theta_{0} .
$$

However, by (5.26) we have,

$$
W_{0}=\phi_{1}\left(h_{m} / z^{\alpha}\right)(1+E) \text { on } F\left(\theta_{0}, \theta_{0}+\varepsilon_{5}\right),
$$


and in view of (5.23), (5.24), (5.28) and Lemma 2.2, we have $W_{0}\left(r e^{i \theta}\right) \rightarrow K_{4} \neq 0$ as $r \rightarrow+\infty$ for $\theta_{0}<\theta<\theta_{0}+\varepsilon_{5}$. This fact, together with (5.31), again provides a contradiction to the same Phragmen-Lindelof principle as in Case I.

This contradiction establishes the theorem.

\section{REFERENCES}

[1] S. BANK, An asymptotic analog of the Fuchs regularity theorem, J. Math. Anal. Appl. 16 (1966), 138-151.

[2] S. BANK, On determining the location of complex zeros of solutions of certain linear differential equations, Ann. Mat. Pura Appl. 151 (1988), 67-96.

[3] S. BANK AND I. LAINe, On the oscillation theory of $f^{\prime \prime}+A f=0$ where $A$ is entire, Trans. Amer. Math. Soc. 273 (1982), 351-363.

[4] S. BANK AND J.K. LANGLEY, On the oscillation of solutions of certain linear differential equations in the complex domain, Proc. Edinburgh Math. Soc. 30 (1987), 455-469.

[5] S. Bank, I. LAine AND J.K. LANGley, On the frequency of zeros of solutions of second-order linear differential equations, Resultate der Math. 10 (1986), 8-24.

[6] W.K. Hayman, Meromorphic Functions, Clarendon Press, Oxford, 1964.

[7] J.K. LANGley, On complex oscillation and a problem of Ozawa, Kodai Math. J. 9 (1986), 430-439.

[8] M. OzAwA, On a solution of $w^{\prime \prime}+e^{-z} w^{\prime}+(a z+b) w=0$, Kodai Math. J. 3 (1980), 295-309.

[9] C. Powder, On the asymptotic behavior of a fundamental set of solutions, Trans. Amer. Math. Soc. 255 (1979), 91-110.

[10] W. StRodT, On the algebraic closure of certain partially ordered fields, Trans. Amer. Math. Soc. 105 (1962), 229-250.

[11] W. Strodt, Contributions to the asymptotic theory of ordinary differential equations in the complex domain, Mem. Amer. Math. Soc., No. 13 (1954).

[12] E.C. Titchmarsh, The Theory of Functions, Oxford University Press, London, 1939.

\footnotetext{
Department of Mathematics

UNIVERSITY OF ILLINOIS

URBANA, IL 61801, USA

Department of Mathematics

University COLLEGE LONDON

LONDON WCIE 6BT, U.K.
} 
